\title{
Prognostic Implication of Tricuspid Regurgitation in STEMI Patients
}

\author{
Ben Sadeh ${ }^{1}$, Tamar Itach $^{2}$, Ilan Merdler ${ }^{1}$, Yogev Peri ${ }^{1}$, Shir Frydman ${ }^{1}$, Samuel Morgan $^{3}$, \\ Aviram Hochstadt ${ }^{1}$, Yotam Pasternak ${ }^{1}$, Yan Topilsky ${ }^{4}$, Shmuel Banai ${ }^{4}$, and Yacov \\ Shacham ${ }^{5}$ \\ ${ }^{1}$ Tel Aviv University Sackler Faculty of Medicine \\ ${ }^{2}$ Tel Aviv Sourasky Medical Center \\ ${ }^{3}$ Tel Aviv University \\ ${ }^{4}$ Tel Aviv Medical Center \\ ${ }^{5}$ Affiliation not available
}

May 18, 2020

\begin{abstract}
Background: Tricuspid regurgitation (TR) is associated with adverse prognosis in various patient populations but currently no data is available about the prevalence and prognostic implication of TR in ST-segment elevation myocardial infarction (STEMI) patients. We investigated the possible implication of TR among STEMI patients. Methods: We conducted a retrospective study of STEMI patients undergoing primary percutaneous coronary intervention (PCI), and its relation to major clinical and echocardiographic parameters. Patients records were assessed for the prevalence and severity of TR, its relation to the clinical profile, key echocardiographic parameters, in-hospital outcomes, and long-term mortality. Patients with previous myocardial infarction or known previous TR were excluded. Results: The study included 1071 STEMI patients admitted between September 2011 and May 2016 (age $61 \pm 13$ years; predominantly male). A total of 205 patients (19\%) had mild TR while another 32 $(3 \%)$ had moderate or greater TR was $3 \%(\mathrm{n}=32)$. Patients with significant TR demonstrated worse echocardiographic parameters, more likely to have in-hospital complications and had higher long-term mortality $(28 \%$ vs. $6 \%$; p $<0.001)$. Following adjustment for significant clinical and echocardiographic parameters, mortality hazard ratio (HR) of at least moderate to severe TR remained significant $(2.44 ; 95 \% \mathrm{CI}, 1.06-5.6 ; \mathrm{P}=.036)$ for patients with moderate-severe TR. Conclusions: Among STEMI patients after primary PCI, the presence of moderate-severe TR was independently associated with adverse outcomes and significantly lower survival rate. Keywords: Tricuspid Regurgitation; ST-segment elevation myocardial infarction; percutaneous coronary intervention; cardiac intensive care unit; cardiac intensive care unit; echocardiography; valvular regurgitation.
\end{abstract}

\section{Introduction}

Tricuspid regurgitation (TR) is a prevalent echocardiographic finding ${ }^{1,2}$, mostly considered as a benign finding and untreated unless associated with severe or major comorbidity such as significant pulmonary hypertension, right or left ventricular dysfunction.

The clinical impact of TR is still under investigation due to variation in the results of previous studies ${ }^{3,4}$. This variation was mainly associated with the heterogenous and numerous comorbidities associated with TR that influenced clinical outcomes, such as low left ventricular ejection fraction (LVEF) ${ }^{5}$, right ventricular $(\mathrm{RV})^{6}$ dilation and dysfunction, and pulmonary artery systolic pressure (PASP) ${ }^{7}$, thus, leading to studies that tried to isolate TR from potential confounders. Recent studies had demonstrated association between severe isolated TR and excess mortality and morbidity in various patient populations ${ }^{2,6,8}$, but lesser degrees of TR were associated as well with poor prognosis, especially in men ${ }^{9}$. Nonetheless, current management 
guidelines of TR patients are ambiguous and most often consider interventional therapy only at the time of mitral or aortic valve surgery ${ }^{5}$.

To date, no study evaluated the prevalence and possible prognostic implication of TR in ST segment elevation (STEMI) patients. In the present study, we investigated the clinical profile, in-hospital outcomes, and longterm mortality associated with the presence of TR in STEMI patients treated with primary percutaneous coronary intervention (PCI).

\section{Methods}

A retrospective, single-center observational study was performed at the Tel-Aviv Sourasky Medical Center, a tertiary referral hospital with a $24 / 7$ primary PCI service, as previously described ${ }^{10}$.

We evaluated 2139 consecutive patients admitted between September 2011 and May 2016 to the cardiac intensive care unit (CICU) with the diagnosis of acute STEMI. We excluded patients with a missing record of tricuspid valve echocardiographic evolution $(\mathrm{n}=847)$, as well as patients with previous myocardial infarction $(\mathrm{n}=196)$ and known previous TR $(\mathrm{n}=25)$. The patient selection process is shown in Figure 1 . The final cohort consisted of 1071 patients whose baseline demographic, cardiovascular history, clinical risk factors, treatment characteristics, and laboratory results were retrieved from their hospital electronic medical files. Diagnosis of STEMI was established in accordance with published guidelines including typical chest pain history, diagnostic electrocardiographic changes, and serial elevation of cardiac biomarkers ${ }^{11}$. Primary PCI was performed in patients with symptoms [?] 12 hours in duration as well as in patients with symptoms lasting 12-24 hours if pain consisted at the time of admission. Symptom duration was defined as the time from symptom onset (usually chest pain or discomfort) to ER/catheterization laboratory admission. Assessment of survival following hospital discharge was determined from computerized records of the population registry bureau. The study protocol was approved by the local institutional ethics committee.

\section{Echocardiography}

All patients underwent a screening echocardiographic examination within 6-72 h of CICU admission. Relevant data were collected from the clinical echocardiographic exam reports. Echocardiography was performed by Philips IE-33 equipped with S5-1 transducers (Philips Healthcare, Andover, MA, USA), and GE Vivid 7 model equipped with M4S transducer.

RV qualitative size and function assessment were based on multiple views of the right ventricle (short-axis parasternal at basal, mid, and apical levels; lower parasternal RV inflow view; apical four-chamber view and, if possible, RV long-axis view; and subcostal short-axis and four-chamber views). Using these multiple views, an integrative qualitative grading was

formulated by the physician responsible for the echocardiographic study. From four-chamber views encompassing the entire right ventricle, end-systolic and end-diastolic RV areas and the tricuspid annulus were measured. Apart from qualitative grading, RV function was evaluated by tricuspid annular plane systolic excursion (TAPSE) ${ }^{12}$.

TR severity was determined using an integrative, semiquantitative approach as recommended by the American Society of Echocardiography ${ }^{13}$.

Regarding the gravity of tricuspid regurgitation, we first assessed the severity of valve regurgitation by evaluating specific signs that would point to either less than mild or severe regurgitation, including color jet area (thin small central vs large $>50 \%$ jet area), vena contracta $(\mathrm{VC})$ width $(<0.2 \mathrm{~cm}$ or [?]7 $\mathrm{mm})$, density of continuous Doppler jet (faint or dense and triangular), hepatic vein flow pattern (systolic dominant vs systolic reversal), trans tricuspid inflow pattern (A-wave dominant or high-velocity E-wave dominant), annular diameter (normal vs dilated annulus with lack of valve coaptation), and RV and right atrial (RA) size (normal vs dilated). If all of the signs and indices were concordant, we defined TR as less than mild or severe. If the signs or values of the qualitative or semiquantitative parameters were in the intermediate 
range between mild and severe, we defined TR as at least moderate to severe if the majority (five or more) of the signs and indices were concordant with severe $\mathrm{TR}^{13}$.

In patients with measurable tricuspid regurgitation jet on Doppler echocardiography peak systolic pulmonary artery pressure (SPAP) was estimated using the modified Bernoulli formula $(4 \times$ TRV2max $)+$ RAP, where TRVmax is the peak systolic tricuspid regurgitation velocity at end expiration, and RAP is the right atrial pressure. Left ventricular (LV) diameters and interventricular septal and posterior wall width were measured from the parasternal short axis by means of a 2-dimensional (2D or a 2D-guided M-mode echocardiogram of the LV at the papillary muscle level using the parasternal short-axis view ${ }^{14}$. LV ejection fraction was calculated by the Biplane method of disks (modified Simpson's rule). Early trans mitral flow velocity (E) was measured in the apical 4-chamber view to provide an estimate of LV diastolic function ${ }^{15}$. Early diastolic mitral annular velocity (e') was measured using spectral tissue Doppler imaging in both septal and lateral positions. The ratio of peak $\mathrm{E}$ to peak e' was calculated (E/e' ratio) from the average of at least 3 cardiac cycles. In patients with atrial fibrillation we have used the average measured from 5-7 cardiac cycles. Left atrial volume was calculated using the biplane area length method at end systole ${ }^{16}$. Cardiac output was calculated as the product of aortic stroke volume and heart rate as demonstrated on pulse wave Doppler. Valvular regurgitation was qualitatively assessed using color Doppler according to the guidelines of the American Society of Echocardiography (normal/trivial $=1$, mild $=2$, moderate $=3$, severe $=4)^{17}$. Diastolic function was assessed by integrating measurements of the mitral inflow, left atrial volume, and Doppler tissue imaging of the mitral annulus using the average annulus velocity, and classified into four categories: normal diastolic function $=0$, impaired relaxation $=1$, pseudo-normal $=2$ and restrictive pattern $=3$, based on recent guidelines ${ }^{15}$. Right atrial (RA) pressure was estimated by the inferior vena cava diameter as well as its response to inspiration as previously described ${ }^{18}$. Briefly, expiratory and inspiratory inferior vena cava (IVC) diameters and percent collapse were measured in subcostal views within $2 \mathrm{~cm}$ of the right atrium. IVC diameter $<2.1 \mathrm{~cm}$ that collapsed $>50 \%$ with a sniff suggested a normal RA pressure (assigned as 5 $\mathrm{mmHg}$ ), whereas an IVC diameter $>2.1 \mathrm{~cm}$ that collapsed $<50 \%$ with a sniff suggested a high RA pressure $(15 \mathrm{mmHg})$. In patients with IVC diameter $<2.1 \mathrm{~cm}$ and no collapse $(<20 \%)$ with a sniff, RA pressure was upgraded to $20 \mathrm{mmHg}$. In indeterminate cases in which the IVC diameter and collapse did not fit this paradigm, secondary indices of elevated RA pressure were integrated. If uncertainty remained, RA pressure was left as intermediate value of $10 \mathrm{mmHg}$.

\section{Statistical analysis}

Patients were divided according to the severity of TR into two groups, those with no or mild TR and those with moderate to severe TR.

All data were summarized and displayed as mean +- standard deviation for continuous variables unless stated otherwise, and as number (percentage) of patients in each group for categorical variables. The $p$ values for the categorical variables were calculated with the chi-square test. Continuous variables were compared using the independent sample t-test or the Mann-Whitney U test. The Kaplan-Meier method and log-rank test were used to evaluate

the association between the severity of TR and survival. To assess if TR grade was

independently associated with outcome, we used multivariate Cox Regression for the primary endpoint (allcause mortality) adjusted for all baseline variables found to be significant in the univariate analysis. A two-tailed p-value of $<0.05$ was considered significant for all analyses. All analyses were performed with the SPSS software (SPSS Inc., Chicago, IL).

\section{Results}

A total of 1071 patients were included in the study, of whom 1039 patients (97\%) with no to mild TR, and $32(3 \%)$ with moderate to severe TR. The mean patient age was $61+-13$ years (range 40 to 91), with a majority of male patients (80\%). Demographic and clinical baseline parameters stratified by severity of TR are shown in Table 1. Patients with moderate to severe TR were significantly older, female and had increased 
prevalence of chronic kidney disease and hypertension.

Key Echocardiographic parameters stratified by severity of TR are shown in Table 2. Patients having moderate to severe TR demonstrated lower ejection fraction, higher septal E/e ratio, SPAP and RA area $(\mathrm{p}<0.05$ for all) compared to No/mild TR group. There was no significant difference in TAPSE between the two groups.

\section{In -hospital and long-term outcome}

Patient with significant TR had higher rate of in-hospital complications (table 3). This included acute kidney injury, heart failure, arrhythmia bleeding and need for mechanical ventilation $(\mathrm{P}<0.05$ for all; table 3$)$.

30 -Day mortality was significantly higher among patients with moderate to severe TR $(4 / 32,12.5 \%)$ compared to patients with no/mild TR $(21 / 1039,2.0 \%$; $<0.001)$.

\section{Long term mortality}

Over a mean period of $2.3+1.5$ years, $71 / 1071(6.6 \%)$ patients of the entire cohort died. Mortality was significantly higher among patients with moderate to severe TR $(9 / 32,28 \%)$ compared to patients with no/mild TR (62/1039, 6\%; p <0.001, Figure 2).

Multivariate Cox hazard analysis for moderate-severe TR adjusted for significant clinical and echocardiographic parameters is shown in Table 4. The mortality HRs of moderate to severe TR (2.44; 95\% CI, 1.06-5.6; $\mathrm{P}=0.03)$ remained significant even after adjustment for gender, family history, hypertension (HTN), estimated glomerular filtration rate $(\mathrm{EGFR})<60, \mathrm{EF}, \mathrm{E} / \mathrm{e}$ ' ratio and SPAP.

\section{Discussion}

The present study demonstrated that among STEMI patient population, the presence of moderate to severe TR was associated with significantly lower survival rate compared to patients with mild/no TR. TR was associated with excess mortality even when adjusted for demographic, clinical and other echocardiography parameters.

To our knowledge, this is the first report to date suggesting a possible prognostic implication of TR in STEMI patients.

TR is a common echocardiographic finding ${ }^{19}$, but has been disregarded due to the credence that it is a clinically insignificant condition. The clinical impact and outcome of TR are difficult to assess, given its heterogeneity and the association with numerous comorbidities. Hence, management guidelines of TR patients remain ambiguous due to conflicting studies results ${ }^{9,20,21}$. Pivotal studies suggested that untreated TR is associated with excess mortality and cardiac events ${ }^{7,21}$. TR had been associated in previous studies with additional cardiovascular outcomes. It has been shown that TR is a common finding in patients with left-sided valvular disease. Significant TR in this circumstance is considered as a late-stage marker and is associated with poor outcome and worse prognosis ${ }^{21-23}$. Therefore, patients undergoing left valve surgery with severe functional TR (FTR) have a class I indication for concomitant tricuspid valve surgery ${ }^{24}$. In patients undergoing transcatheter aortic valve replacement (TAVR) the impact of preoperative significant TR was associated with almost a 2 -fold increase in 2-year mortality ${ }^{23}$.

TR is also common among patients with chronic heart failure (CHF), due to the pathophysiology of CHF resulting in right ventricular dilatation, and the development of FTR which, consequently, generating further right ventricular dilatation and worsening of $\mathrm{TR}^{25}$. Studies indicate a strong impact of $\mathrm{TR}$ on the clinical outcome in CHF patients, where TR was significantly related to mortality ${ }^{26}$.

Recent studies had demonstrated that moderate-severe TR is associated with poor outcome, even in the absence of left ventricular dysfunction or pulmonary hypertension ${ }^{3,7,9}$, implying that tricuspid valve repair or replacement may lead to a survival benefit. However, to date, TR patients are rarely referred for isolated surgical tricuspid valve repair, and these are mostly performed during other planned cardiac surgery ${ }^{5,27,28}$. 
In the era where percutaneous repair procedures are on the rise, more research on percutaneous approaches for TR is needed ${ }^{28}$.

Limited data exist on the prevalence and prognostic value of significant TR in STEMI patients undergoing PCI. In the setting of acute occlusion of the right coronary artery leading to inferior MI, RV involvement, and concomitant severe TR, tricuspid papillary muscle rupture (PMR) had been reported as a rare complication 29,30 .

The present study provides, for the first time, evidence that moderate to severe TR can serve as a possible prognostic marker among STEMI patients. We found that among STEMI patients undergoing primary PCI, with no previous TR, the prevalence for developing moderate to severe TR was $2.7 \%$. These patients suffered more in-hospital complications and worse long-term outcomes. These results imply that in patients developing moderate to severe TR, additional follow up after PCI is needed. Once released from hospital these patients should be followed by a cardiologist, undergo an additional echocardiographic exam to track progression of TR severity and possibly electrocardiogram exam due to a high prevalence of arrythmias. An extra emphasis should be placed on balancing of cardiovascular risk factors for these patients.

Though the reason for higher mortality among STEMI patients with significant TR is yet unclear, we postulate that the presence of TR after STEMI could be a marker of decreased RV function and contractility. It has been shown that the presence of severe TR can be attributed to RV akinesis in the settings of inferior MI or to ischemic impairment of the tricuspid valve ${ }^{29}$. Moreover, increasing severity of TR is allied with RV dilation, dysfunction and elevated right atrial pressure, therefore leading to a worse outcomes ${ }^{25}$. In addition, the association between enlarged RV and increased mortality was demonstrated in previous studies ${ }^{31,32}$, elucidating that RV function after STEMI has important prognostic implications. Nevertheless, from our understanding the RV function influence on outcomes only partly explains the association between significant TR among STEMI patients and mortality, therefore, additional research is needed in order to illuminate the matter.

We acknowledge several important limitations of our study. This was a single-center retrospective and nonrandomized observational study; because of its retrospective nature, the study was subjected to selection bias, and therefore the results point toward association, and not cause and effect.

The study included only patients with first MI who were undergoing primary PCI and with no previous TR. Therefore, the results cannot be generalized to all STEMI patients with TR. The group with moderate to severe TR was small, patients were significantly older, female and with CKD. We attempted to adjust for confounding factors using the multivariate Cox hazard model, however, most of the study population were men, hence the data may not be applicable to female patients. Finally, data were collected retrospectively from echocardiographic reports who were recorded and analyzed by different sonographers. An echocardiographic exam is highly operator dependent which may be subjective, even though it was determined by echocardiography experts.

\section{Conclusions:}

Among STEMI patients after primary PCI, those who develop moderate to severe TR have a significantly lower survival rate. Nevertheless, our results should be interpreted with caution and more research is needed in the future with prospective randomized trials.

The authors declare that they have no conflict of interest.

\section{References}

1. Singh JP, Evans JC, Levy D, et al. Prevalence and clinical determinants of mitral, tricuspid, and aortic regurgitation (The Framingham Heart Study). Am J Cardiol . 1999;83(6):897-902. doi:10.1016/S00029149(98)01064-9

2. Ghadrdoost B, Kyavar M, Haghighat-Talab A, et al. Impact of severe tricuspid regurgitation on long term survival. Res Cardiovasc Med . 2015;2(3):121. doi:10.5812/cardiovascmed.11281 
3. Bar N, Schwartz LA, Biner S, et al. Clinical Outcome of Isolated Tricuspid Regurgitation in Patients with Preserved Left Ventricular Ejection Fraction and Pulmonary Hypertension. J Am Soc Echocardiogr . 2018;31(1):34-41. doi:10.1016/j.echo.2017.09.010

4. Topilsky Y, Inojosa JM, Benfari G, et al. Clinical presentation and outcome of tricuspid regurgitation in patients with systolic dysfunction. Eur Heart J . 2018;39(39):3584-3592. doi:10.1093/eurheartj/ehy434

5. Nishimura RA, Otto CM, Bonow RO, et al. 2014 AHA/ACC Guideline for the Management of Patients With Valvular Heart Disease: Executive Summary. Circulation . 2014;129(23):2440-2492. doi:10.1161/CIR.0000000000000029

6. Schwartz LA, Rozenbaum Z, Ghantous E, et al. Impact of Right Ventricular Dysfunction and Tricuspid Regurgitation on Outcomes in Patients Undergoing Transcatheter Aortic Valve Replacement. J Am Soc Echocardiogr . 2017;30(1):36-46. doi:10.1016/j.echo.2016.08.016

7. Topilsky Y, Nkomo VT, Vatury O, et al. Clinical Outcome of Isolated Tricuspid Regurgitation. JACC Cardiovasc Imaging . 2014;7(12):1185-1194. doi:10.1016/j.jcmg.2014.07.018

8. Sagie A, Schwammenthal E, Newell JB, et al. Significant tricuspid regurgitation is a marker for adverse outcome in patients undergoing percutaneous ballon mitral valvuloplasty. J Am Coll Cardiol . 1994;24(3):696-702. doi:10.1016/0735-1097(94)90017-5

9. Nath J, Foster E, Heidenreich PA. Impact of Tricuspid Regurgitation on Long-Term Survival. J Am Coll Cardiol . 2004;43(3):405-409. doi:10.1016/j.jacc.2003.09.036

10. Arbel Y, Shacham Y, Finkelstein A, et al. Red Blood Cell Distribution Width (RDW) and longterm survival in patients with ST Elevation Myocardial Infarction. Thromb Res . 2014;134(5):976-979. doi:10.1016/j.thromres.2014.08.016

11. O'Gara PT, Kushner FG, Ascheim DD, et al. 2013 ACCF/AHA Guideline for the Management of ST-Elevation Myocardial Infarction: Executive Summary. Circulation . 2013;127(4):529-555. doi:10.1161/CIR.0b013e3182742c84

12. Arkles JS, Opotowsky AR, Ojeda J, et al. Shape of the right ventricular Doppler envelope predicts hemodynamics and right heart function in pulmonary hypertension. Am J Respir Crit Care Med . 2011;183(2):268-276. doi:10.1164/rccm.201004-0601OC

13. Zoghbi WA, Adams D, Bonow RO, et al. Recommendations for Noninvasive Evaluation of Native Valvular Regurgitation: A Report from the American Society of Echocardiography Developed in Collaboration with the Society for Cardiovascular Magnetic Resonance. J Am Soc Echocardiogr . 2017;30(4):303-371. doi:10.1016/j.echo.2017.01.007

14. Lang RM, Badano LP, Mor-Avi V, et al. Recommendations for cardiac chamber quantification by echocardiography in adults: An update from the American society of echocardiography and the European association of cardiovascular imaging. Eur Heart J Cardiovasc Imaging . 2015;16(3):233-271. doi:10.1093/ehjci/jev014

15. Nagueh SF, Smiseth OA, Appleton CP, et al. Recommendations for the evaluation of left ventricular diastolic function by echocardiography: An update from the American society of echocardiography and the European association of cardiovascular imaging. Eur Heart J Cardiovasc Imaging . 2016;17(12):1321-1360. doi:10.1093/ehjci/jew082

16. LANG R, BIERIG M, DEVEREUX R, et al. Recommendations for chamber quantification. Eur $J$ Echocardiogr . 2006;7(2):79-108. doi:10.1016/j.euje.2005.12.014

17. ZOGHBI W, Kraft CD, Levine RA, et al. Recommendations for evaluation of the severity of native valvular regurgitation with two-dimensional and doppler echocardiography. J Am Soc Echocardiogr . 2003;16(7):777-802. doi:10.1016/S0894-7317(03)00335-3 
18. Kircher BJ, Himelman RB, Schiller NB. Noninvasive estimation of right atrial pressure from the inspiratory collapse of the inferior vena cava. Am J Cardiol . 1990;66(4):493-496. doi:10.1016/0002-9149(90)90711-9

19. Singh JP, Evans JC, Levy D, al. et. Prevalence and clinical determinates of mitral, tricuspid, and aortic regurgitation. Am J Cardiol . 1999.

20. Messika-Zeitoun D, Thomson H, Bellamy M, et al. Medical and surgical outcome of tricuspid regurgitation caused by flail leaflets. J Thorac Cardiovasc Surg . 2004. doi:10.1016/j.jtcvs.2004.01.035

21. Sagie A, Schwammenthal E, Newell JB, et al. Significant tricuspid regurgitation is a marker for adverse outcome in patients undergoing percutaneous ballon mitral valvuloplasty. J Am Coll Cardiol . 1994. doi:10.1016/0735-1097(94)90017-5

22. Topilsky Y, Nkomo VT, Vatury O, et al. Clinical outcome of isolated tricuspid regurgitation. JACC Cardiovasc Imaging . 2014. doi:10.1016/j.jcmg.2014.07.018

23. Barbanti M, Binder RK, Dvir D, et al. Prevalence and Impact of Preoperative Moderate / Severe Tricuspid Regurgitation on Patients Undergoing Transcatheter Aortic Valve Replacement. doi:10.1002/ccd.25512

24. Mahesh B, Wells F, Nashef S, Nair S. Role of concomitant tricuspid surgery in moderate functional tricuspid regurgitation in patients undergoing left heart valve surgery. Eur J Cardiothorac Surg . 2013;43(1):2-8. doi:10.1093/ejcts/ezs441

25. Neuhold S, Huelsmann M, Pernicka E, et al. Impact of tricuspid regurgitation on survival in patients with chronic heart failure : unexpected findings of a long-term observational study. 2013:844-852. doi:10.1093/eurheartj/ehs465

26. Koelling TM, Aaronson KD, Cody RJ, Bach DS, Armstrong WF. Prognostic significance of mitral regurgitation and tricuspid regurgitation in patients with left ventricular systolic dysfunction. Am Heart $J$ . 2002;144(3):524-529. doi:10.1067/mhj.2002.123575

27. Lee J-W, Song J-M, Park JP, Lee JW, Kang D-H, Song J-K. Long-term prognosis of isolated significant tricuspid regurgitation. Circ J . 2010.

28. Rogers JH, Bolling SF. The tricuspid valve: Current perspective and evolving management of tricuspid regurgitation. Circulation . 2009. doi:10.1161/CIRCULATIONAHA.108.842773

29. Walker JR, Mousavi N, Horlick E, Seifer C, Jassal DS. Tricuspid valvular papillary muscle rupture with intractable hypoxia: a rare complication post MI. J Am Soc Echocardiogr . 2009;22(7):863.e1-3. doi:10.1016/j.echo.2009.02.021

30. Goldstein JA, Oak R. Pathophysiology and Management of Right Heart Ischemia. J Am Coll Cardiol . 2002;40(5):841-853. doi:10.1016/S0735-1097(02)02048-X

31. Lewis JF, Webber JD, Sutton LL, Chesoni S, Curry CL. Discordance in degree of right and left ventricular dilation in patients with dilated cardiomyopathy: recognition and clinical implications. J Am Coll Cardiol . 1993;21(3):649-654. doi:10.1016/0735-1097(93)90097-k

32. Hoogslag GE, Haeck MLA, Velders MA, et al. Determinants of right ventricular remodeling following ST-segment elevation myocardial infarction. Am J Cardiol . 2014;114(10):1490-1496. doi:10.1016/j.amjcard.2014.08.006

Figure 1. Patient selection process. STEMI, ST-segment elevation myocardial infarction; TR,

Tricuspid Regurgitation; Mod, moderate.

Figure 2. Cox regression curve for overall survival of patients. TR, Tricuspid Regurgitation.

\section{Hosted file}


Prognostic Implication of TR in STEMI Patients- tables.docx available at https://authorea. com/users/322606/articles/451568-prognostic-implication-of-tricuspid-regurgitation-instemi-patients

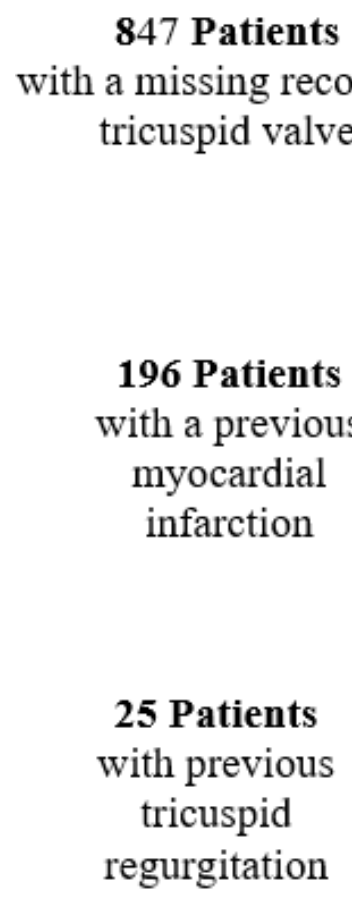

\section{Patients with Acute STEMI}

1292 Patients

196 Patients with a previous myocardial infarction

\section{Patients}

with previous regurgitation

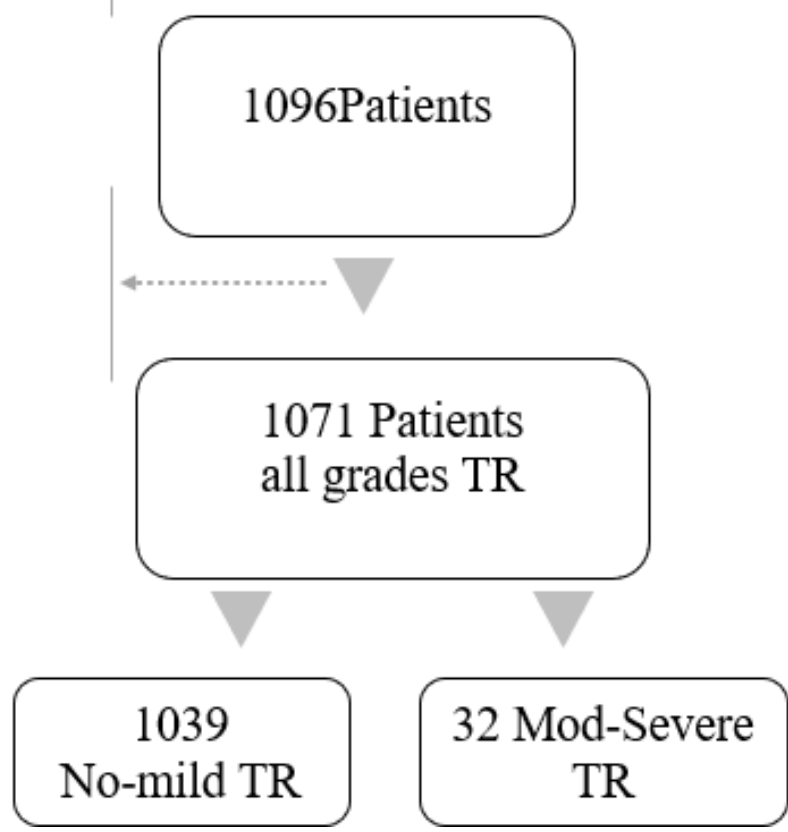




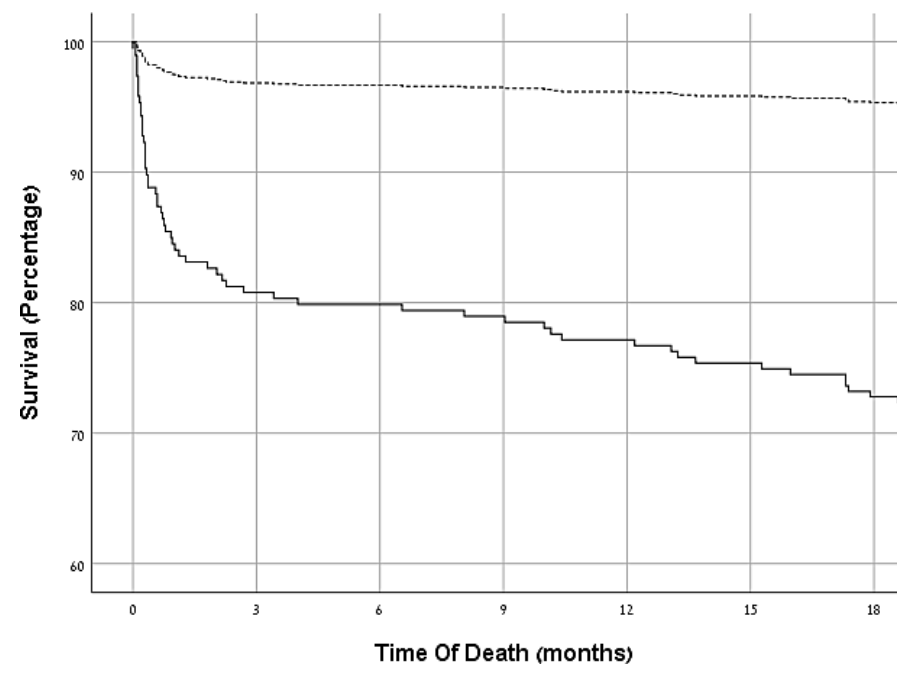

TR none-mild vs.

moderate-severe

...- None-Mild

$\neg$ Moderate-Severe 